\title{
Article
}

\section{On Testing the Adequacy of the Inverse Gaussian Distribution}

\author{
James S. Allison ${ }^{1}$, Steffen Betsch ${ }^{2}$, Bruno Ebner ${ }^{2, *}$ and Jaco Visagie ${ }^{1}$ \\ 1 School of Mathematical and Statistical Sciences, North-West University, Potchefstroom 2531, South Africa; \\ james.allison@nwu.ac.za (J.S.A.); jaco.visagie@nwu.ac.za (J.V.) \\ 2 Institute of Stochastics, Karlsruhe Institute of Technology (KIT), 76131 Karlsruhe, Germany; \\ steffen.betsch@kit.edu \\ * Correspondence: bruno.ebner@kit.edu
}

Citation: Allison, J.S.; Betsch, S.; Ebner, B.; Visagie, J. On Testing the Adequacy of the Inverse Gaussian Distribution. Mathematics 2022, 10, 350. https://doi.org/10.3390/ math10030350

Academic Editor: Stefano Bonnini

Received: 13 December 2021

Accepted: 14 January 2022

Published: 24 January 2022

Publisher's Note: MDPI stays neutral with regard to jurisdictional claims in published maps and institutional affiliations.

Copyright: () 2022 by the authors Licensee MDPI, Basel, Switzerland. This article is an open access article distributed under the terms and conditions of the Creative Commons Attribution (CC BY) license (https:// creativecommons.org/licenses/by/ $4.0 /)$.

\begin{abstract}
We propose a new class of goodness-of-fit tests for the inverse Gaussian distribution based on a characterization of the cumulative distribution function (CDF). The new tests are of weighted $L^{2}$ type depending on a tuning parameter. We develop the asymptotic theory under the null hypothesis and under a broad class of alternative distributions. These results guarantee that the parametric bootstrap procedure, which we employ to implement the test, is asymptotically valid and that the whole test procedure is consistent. A comparative simulation study for finite sample sizes shows that the new procedure is competitive to classical and recent tests, outperforming these other methods almost uniformly over a large set of alternative distributions. The use of the newly proposed test is illustrated with two observed data sets.
\end{abstract}

Keywords: goodness-of-fit tests; inverse gaussian distribution; parametric bootstrap; stein-type characterization; warp-speed bootstrap

\section{Introduction}

The inverse Gaussian distribution (also known as the Wald distribution) was first heuristically observed by [1], and derived by [2] as the distribution of the first passage time of Brownian motion with drift, see [3] for a historical summary. In the statistical literature the usual parametrization of the inverse Gaussian law $\operatorname{IG}(\mu, \lambda), \mu, \lambda>0$, follows the representation of $[4,5]$, namely the density is given by

$$
f(x ; \mu, \lambda)=\sqrt{\frac{\lambda}{2 \pi x^{3}}} \exp \left(-\frac{\lambda(x-\mu)^{2}}{2 \mu^{2} x}\right), \quad x>0,
$$

and $f(x ; \mu, \lambda)=0$ for $x \leq 0$. Applications involving the inverse Gaussian family of distributions, $\mathcal{I} \mathcal{G}=\{\operatorname{IG}(\mu, \lambda): \mu, \lambda>0\}$, cover topics such as stock market prices, biology, hydrology, reliability, Lévy processes, and generalized linear models, as witnessed by the monographs especially dedicated to this family of distributions, see [3,6,7].

The first step in serious statistical inference with this distribution is to test the fit of data to the family $\mathcal{I} \mathcal{G}$. To be specific, let $X, X_{1}, X_{2}, \ldots$ be positive, independent and identically distributed (i.i.d.) random variables defined on a common probability space $(\Omega, \mathcal{A}, \mathbb{P})$. Writing $\mathbb{P}^{X}$ for the distribution of $X$, we intend to test the composite hypothesis

$$
H_{0}: \mathbb{P}^{X} \in \mathcal{I G}
$$

against general alternatives. This testing problem has been considered in the statistical literature. The methods by [8,9] are based on a characterization of the $\mathcal{I} \mathcal{G}$ family by an independence property. In [10] a connection to the so-called random walk distribution is used, and [11] proposes exact tests based on the empirical distribution function of transformations characterizing the inverse Gaussian law, which are corrected in [12]. The 
author of [13] use a differential equation that characterizes the Laplace transform of the $\mathcal{I} \mathcal{G}$ family as well as an $L^{2}$-distance test, both using the empirical Laplace transform. In [14] an empirical version of the standardized form of the cumulant-generating function is used, in [15] an empirical likelihood test based on densities for $\mathcal{I} \mathcal{G}$ is proposed, and in [16] a variance ratio test of fit is considered. Finally, the authors of [17] tackle the testing problem for the generalized inverse Gaussian family exploiting the ULAN property in connection to Le Cam theory. Although the testing problem is derived for a wider class of distributions, it still applies for testing (2) when it is restricted to the special case $p_{0}=-\frac{1}{2}$ in the authors notation; see Section 4 of the cited article. A comparative simulation study is provided by [18].

It is a common approach to exploit distributional characterizations to propose goodness-offit testing procedures; for an overview see [19]. As demonstrated by the list above, there are numerous characterizations of the inverse Gaussian distribution, including characterizing properties based on independence, constancy of regression of suitable functions on the sum of identically distributed random variables, random continued fractions, or on the relationship between $\mathbb{E}[X]$ and $\mathbb{E}\left[X^{-1}\right]$. For details, see Section 3 of [3], and Section 2.5 of [20], and for an introduction to characterizations for other distributions, see [21,22]. A recent characterization identity is given in Example 5 of [23], which reads as follows.

Theorem 1. Let $X: \Omega \rightarrow(0, \infty)$ be a random variable with distribution function $F, \mathbb{E}[X]<\infty$ and $\mathbb{E}\left[X^{-1}\right]<\infty$. Then $X$ has the inverse Gaussian distribution $\operatorname{IG}(1, \varphi)$ if, and only if,

$$
\mathbb{E}\left[\frac{1}{2}\left(\varphi+\frac{3}{X}-\frac{\varphi}{X^{2}}\right) \min \{X, t\}\right]=F(t), \quad t>0 .
$$

Note that $X \sim \operatorname{IG}(\mu, \lambda)$ if, and only if, $\frac{X}{\mu} \sim \operatorname{IG}(1, \lambda / \mu)$ and therefore the family $\mathcal{I} \mathcal{G}$ is closed under scale transformations. Since the characterization is directly related to the theory of Stein characterizations (for details on Stein operators, see [24]), we refer to the corresponding characterization of the generalized inverse Gaussian distribution, as in Theorem 3.2 of [20], and to the connection with the Stein operator for the special case $(p, a, b)=\left(-1 / 2, \lambda / \mu^{2}, \lambda\right)$, using the authors' notation.

Our novel testing procedure is motivated by Theorem 1: We estimate both sides of (3) by their empirical counterparts, then calculate a weighted $L^{2}$-distance of the difference. These $L^{2}$-type statistics are widely used in goodness-of-fit testing; see [25]. In this spirit, we propose the statistic

$$
T_{n}=n \int_{0}^{\infty}\left|\frac{1}{2 n} \sum_{j=1}^{n} \Delta_{n, j} \min \left\{Y_{n, j}, t\right\}-\frac{1}{n} \sum_{j=1}^{n} \mathbb{1}\left\{Y_{n, j} \leq t\right\}\right|^{2} w(t) \mathrm{d} t
$$

with $\Delta_{n, j}=\widehat{\varphi}_{n}+\frac{3}{Y_{n, j}}-\frac{\widehat{\varphi}_{n}}{Y_{n, j}^{2}}$ and $Y_{n, j}=\frac{X_{j}}{\widehat{\mu}_{n}}$, where $\widehat{\mu}_{n}, \widehat{\lambda}_{n}$, are consistent estimators of $\mu, \lambda$, respectively, and $\widehat{\varphi}_{n}=\widehat{\lambda}_{n} / \widehat{\mu}_{n}$. The continuous function $w(t)$ puts positive weight on $(0, \infty)$, and satisfies

$$
\int_{0}^{\infty}\left(t^{2}+1\right) w(t) \mathrm{d} t<\infty \text { and } n \int_{0}^{\infty}\left|w\left(\hat{\lambda}_{n}^{-1} s\right)-w(s)\right|^{3}(w(s))^{-2} \mathrm{~d} s \stackrel{\mathbb{P}}{\longrightarrow} 0,
$$

where $\stackrel{\mathbb{P}}{\longrightarrow}$ denotes convergence in probability (as $n \rightarrow \infty)$. Note that when suitable weight functions are chosen, the calculation of $T_{n}$ does not require numerical integration; see Section 5.1. In particular, we use the weights $w_{a}(t)=e^{-a t}$ and $\widetilde{w}_{a}(t)=e^{-a t^{2}}, t>0$, with a positive tuning parameter $a>0$. Both satisfy the conditions in (4). A proof of this fact is given by [26] for the first weight function, and the argument for the second weight is very similar, so we do not discuss it here. For a discussion of the use of weight functions in goodness-of-fit testing, see for example $[27,28]$. Since $\mathcal{I} \mathcal{G}$ is scale invariant, the test should 
reflect this property. Thus, we only consider scale-equivariant estimators $\widehat{\mu}_{n}$ of $\mu$, i.e., we have

$$
\widehat{\mu}_{n}\left(\beta X_{1}, \ldots, \beta X_{n}\right)=\beta \widehat{\mu}_{n}\left(X_{1}, \ldots, X_{n}\right), \quad \beta>0 .
$$

Likewise, let $\hat{\lambda}_{n}$ be an estimator of $\lambda$ which is scale-equivariant and thus ensures that $\widehat{\varphi}_{n}$ is scale invariant, i.e.,

$$
\widehat{\varphi}_{n}\left(\beta X_{1}, \ldots, \beta X_{n}\right)=\widehat{\varphi}_{n}\left(X_{1}, \ldots, X_{n}\right), \quad \beta>0 .
$$

With this type of estimator it is straightforward to show that $T_{n}$ is invariant under scale transformations of the data, as it depends on (the scale invariant) $Y_{n, j}, j=1, \ldots, n$, and $\widehat{\varphi}_{n}$ only. Rejection of $H_{0}$ in (2) is for large values of $T_{n}$.

The paper is structured as follows. In Section 2 we present two estimation procedures for the parameters in the $\mathcal{I} \mathcal{G}$-family as well as asymptotic representations of the estimators needed in the subsequent theory. Section 3 provides theoretical asymptotic results under the null hypothesis, summarizes the behavior under contiguous alternatives, and contains a limit result under a large class of alternative distributions. In Section 4 we explain the implementation of the test via a parametric bootstrap procedure, and we prove consistency of this bootstrap-based testing method. We end the article with a Monte Carlo power study and an application to observed data examples in Section 5, and draw conclusions in Section 6.

\section{Estimation of the Parameters and Asymptotic Representations}

In this section, we consider two suitable estimators which satisfy the requirement of scale equivariance. The estimators in use are the maximum likelihood (ML) and the moment (MO) estimators. For details of the estimation procedures, we refer to [29], Chapter 15, and [3], Chapter 6. To account for the bootstrap procedure used to obtain critical values, we later study the asymptotic behavior of $T_{n}$ under a triangular array $X_{n, 1}, \ldots, X_{n, n}$ of rowwise i.i.d. random variables, where $X_{n, 1} \sim \operatorname{IG}\left(1, \varphi_{n}\right)$ for a sequence of positive numbers $\left(\varphi_{n}\right)_{n \in \mathbb{N}}$ with $\lim _{n \rightarrow \infty} \varphi_{n}=\varphi>0$. Notice that in the following we assume, without loss of generality and with respect to the scale invariance of the test statistic, that $\mu=1$. We write $o_{\mathbb{P}}(1)$ and $O_{\mathbb{P}}(1)$ for (real-valued) random variables that converge to 0 in probability or that are bounded in probability, respectively. For both estimation methods, we need expansions of the form

$$
\begin{aligned}
\sqrt{n}\left(\widehat{\mu}_{n}-1\right) & =\frac{1}{\sqrt{n}} \sum_{j=1}^{n} \Psi_{1}\left(X_{n, j}\right)+\varepsilon_{n, 1}, \\
\sqrt{n}\left(\widehat{\lambda}_{n}-\varphi_{n}\right) & =\frac{1}{\sqrt{n}} \sum_{j=1}^{n} \Psi_{2}\left(X_{n, j}, \varphi_{n}\right)+\varepsilon_{n, 2},
\end{aligned}
$$

where $\varepsilon_{n, j}=o_{\mathbb{P}}(1), j=1,2$, and $\Psi_{j}$ are measurable functions such that the random variables $\Psi_{1}\left(X_{n, 1}\right)$ and $\Psi_{2}\left(X_{n, 1}, \varphi_{n}\right)$ are centered with existing second moment, and

$$
\begin{aligned}
\lim _{n \rightarrow \infty} \mathbb{E}\left[\left(\Psi_{1}\left(X_{n, 1}\right)\right)^{2}\right] & =\mathbb{E}\left[\left(\Psi_{1}(X)\right)^{2}\right], \\
\lim _{n \rightarrow \infty} \mathbb{E}\left[\left(\Psi_{2}\left(X_{n, 1}, \varphi_{n}\right)\right)^{2}\right] & =\mathbb{E}\left[\left(\Psi_{2}(X, \varphi)\right)^{2}\right],
\end{aligned}
$$

where $X \sim \operatorname{IG}(1, \varphi)$. In principle, any other estimation method that gives scale-equivariant estimators that allow for such asymptotic expansions can be considered as well, but we focus on the following estimators. 
1. Maximum likelihood estimators: Standard calculations show that

$$
\widehat{\mu}_{n}=\frac{1}{n} \sum_{j=1}^{n} X_{n, j}=\bar{X}_{n} \quad \text { and } \quad \widehat{\lambda}_{n}=\left(\frac{1}{n} \sum_{j=1}^{n}\left(\frac{1}{X_{n, j}}-\frac{1}{\bar{X}_{n}}\right)\right)^{-1}
$$

are the ML estimators of $\mu$ and $\lambda$. The asymptotic expansions as in (5) are derived as

$$
\begin{aligned}
& \sqrt{n}\left(\widehat{\mu}_{n}-1\right)=\frac{1}{\sqrt{n}} \sum_{j=1}^{n}\left(X_{n, j}-1\right) \text { and } \\
& \sqrt{n}\left(\widehat{\lambda}_{n}-\varphi_{n}\right)=\frac{1}{\sqrt{n}} \sum_{j=1}^{n}\left(-\frac{\varphi_{n}^{2}}{X_{n, j}}-\varphi_{n}^{2} X_{n, j}+2 \varphi_{n}^{2}+\varphi_{n}\right)+o_{\mathbb{P}}(1) .
\end{aligned}
$$

2. Moment estimators: The moment estimators based on the first two moments of the inverse Gaussian distribution are

$$
\widehat{\mu}_{n}=\bar{X}_{n} \text { and } \quad \widehat{\lambda}_{n}=\frac{\widehat{\mu}_{n}^{3}}{\frac{1}{n} \sum_{j=1}^{n} X_{n, j}^{2}-\widehat{\mu}_{n}^{2}},
$$

with the same asymptotic expansion for $\widehat{\mu}_{n}$ as in the case of ML estimation, and

$$
\begin{aligned}
\sqrt{n}\left(\widehat{\lambda}_{n}-\varphi_{n}\right) & =\frac{1}{\sqrt{n}} \sum_{j=1}^{n}\left(-\varphi_{n}^{2} X_{n, j}^{2}+\left(3 \varphi_{n}+2 \varphi_{n}^{2}\right) X_{n, j}-\varphi_{n}\left(2+\varphi_{n}\right)\right) \\
& +o_{\mathbb{P}}(1) .
\end{aligned}
$$

Remark 1. Note that if $X, X_{1}, X_{2}, \ldots$ are any i.i.d. positive random variables such that $\mathbb{E}[X+$ $\left.X^{-1}\right]<\infty$ (and $\mathbb{E}\left[X^{2}\right]<\infty$ in the case of the moment estimators), the definitions and asymptotic expansions above remain essentially the same, only $X_{n, j}$ is replaced by $X_{j}$, and $\varphi_{n}$ by $\varphi$ (with $\varphi$ defined as below). To see that the asymptotic expansions continue to hold, notice that by the scale invariance of our test statistic, we may assume that $\mathbb{E}[X]=1$, hence $\widehat{\mu}_{n} \rightarrow 1 \mathbb{P}$-almost surely (a.s.) and $\Psi_{1}\left(X_{j}\right)=X_{j}-1$ is centered. Similarly, using that

$$
\begin{aligned}
& \widehat{\lambda}_{n}=\left(\frac{1}{n} \sum_{j=1}^{n}\left(\frac{1}{X_{n, j}}-\frac{1}{\bar{X}_{n}}\right)\right)^{-1} \longrightarrow\left(\mathbb{E} X^{-1}-1\right)^{-1}=: \varphi \\
& \text { or } \widehat{\lambda}_{n}=\frac{\widehat{\mu}_{n}^{3}}{\frac{1}{n} \sum_{j=1}^{n} X_{n, j}^{2}-\widehat{\mu}_{n}^{2}} \longrightarrow\left(\mathbb{E} X^{2}-1\right)^{-1}=: \varphi
\end{aligned}
$$

$\mathbb{P}$-a.s., as $n \rightarrow \infty$, for the $M L$ or $M O$ estimator, respectively, the corresponding expansions are seen to remain valid with the mentioned replacements.

For the ML estimators, also note that since the $\mathcal{I} \mathcal{G}$ family is a 2-parameter general exponential family, the statistic $\left(\bar{X}_{n}, \sum_{j=1}^{n}\left(X_{j}^{-1}-\bar{X}_{n}^{-1}\right)\right)$ is minimal sufficient and complete. An application of the Lehmann-Scheffé theorem shows that $\widehat{\mu}_{n}$ and $\frac{n-1}{n} \widehat{\lambda}_{n}$ are uniformly minimum variance unbiased estimators; see [3]. Note that $\frac{n-1}{n} \widehat{\lambda}_{n}$ is the same estimator as the one considered by [30].

Remark 2. Although we do not use Bayes estimation in this paper, some remarks and references are in order. The authors of [31] study Bayesian estimation of the parameters of the inverse Gaussian distribution and use Markov chain Monte Carlo techniques to implement these estimates. In [32] some inferential results for the inverse Gaussian distribution are derived by considering a proper prior distribution, while [33] performed Bayesian inference for the parameters of this distribution and compared the results with the classical maximum likelihood estimators. 


\section{The Limit Null Distribution, Contiguous Alternatives, and a Functional Law of Large Numbers}

This section is dedicated to asymptotic results with the goal of providing a consistency result for our new test in a realistic setting, meaning a theoretical setting which rigorously takes into account the parametric bootstrap procedure that needs to be applied to implement the test. The techniques used below are similar to those used in [26] in the context of the gamma distribution. We thus omit the details and focus on the central findings.

A suitable setting to derive asymptotic results for the test statistic is based on $L^{2}=L^{2}((0, \infty), w(t) \mathrm{d} t)$, the Hilbert space of Borel-measurable functions $g:(0, \infty) \rightarrow$ $(0, \infty)$ satisfying $\int_{0}^{\infty}(g(t))^{2} w(t) \mathrm{d} t<\infty$. The norm in $L^{2}$ is denoted by $\|g\|_{L^{2}}=\left(\int_{0}^{\infty}(g(t))^{2} w(t) \mathrm{d} t\right)^{1 / 2}$, for $g \in L^{2}$. We consider the triangular array $X_{n, 1}, \ldots, X_{n, n}$ from Section 2. In particular, recall that $X_{n, 1} \sim \operatorname{IG}\left(1, \varphi_{n}\right)$ with $\varphi_{n} \rightarrow \varphi>0$ as $n \rightarrow \infty$, and define

$$
V_{n}(t)=\frac{1}{\sqrt{n}} \sum_{j=1}^{n}\left[\frac{1}{2}\left(\frac{\widehat{\lambda}_{n}}{\widehat{\mu}_{n}^{2}}+\frac{3}{X_{n, j}}-\frac{\widehat{\lambda}_{n}}{X_{n, j}^{2}}\right) \min \left\{X_{n, j}, t\right\}-\mathbb{1}\left\{X_{n, j} \leq t\right\}\right],
$$

$t>0$, for one of the two types of estimators $\widehat{\mu}_{n}$ and $\widehat{\lambda}_{n}$ as in Section 2 . Note that $V_{n}(\cdot)$ is a random element of $L^{2}$, and after a simple change of variable we have

$$
T_{n}=\frac{1}{\widehat{\mu}_{n}} \int_{0}^{\infty}\left(V_{n}(t)\right)^{2} w\left(\frac{t}{\widehat{\mu}_{n}}\right) \mathrm{d} t \text {. }
$$

With the assumptions on the weight function in (4), it is possible to write $T_{n}=$ $\frac{1}{\hat{\mu}_{n}}\left\|V_{n}\right\|_{L^{2}}^{2}+o_{\mathbb{P}}(1)$ and hence express the test statistic in terms of the Hilbert space norm. Retracing and adapting the steps from the proof of Theorem 3 of [26], applying the Hilbert space central limit theorem for triangular arrays due to [34], and facilitating the asymptotic representations of the estimators from (5) allows us to calculate the limit distribution of the test statistic under the hypothesis. Writing $\stackrel{\mathcal{D}}{\longrightarrow}$ for convergence in distribution, the specific result reads as follows.

Theorem 2. Under the standing assumptions there exists a centered Gaussian element, $\mathcal{W}$, of $L^{2}$ with covariance kernel

$$
\mathcal{K}_{\varphi}(s, t)=\mathbb{E}\left[g_{\varphi}(s, X) g_{\varphi}(t, X)\right], \quad s, t>0,
$$

where

$$
\begin{array}{r}
g_{\varphi}(s, x)=\frac{1}{2}\left(\varphi+\frac{3}{x}-\frac{\varphi}{x^{2}}\right) \\
\min \{x, s\}-\mathbb{1}\{x \leq s\}-\varphi \Psi_{1}(x) \mathbb{E}[\min \{X, s\}] \\
+\frac{1}{2} \Psi_{2}(x, \varphi) \mathbb{E}\left[\min \{X, s\}\left(1-X^{-2}\right)\right], \quad x, s>0,
\end{array}
$$

such that $T_{n} \stackrel{\mathcal{D}}{\longrightarrow}\|\mathcal{W}\|_{L^{2}}^{2}$, as $n \rightarrow \infty$.

With the limit null distribution from Theorem 2 at hand, Theorem 4 of [26] can be adapted to obtain the asymptotic behavior of the new test under contiguous alternatives to a fixed representative of $\mathcal{I} \mathcal{G}$. To this end, let $f_{0}$ denote the density of the $\operatorname{IG}(1, \varphi)$ distribution for a fixed $\varphi>0$, and let $c:(0, \infty) \rightarrow \mathbb{R}$ be a measurable, bounded function satisfying $\int_{0}^{\infty} c(x) f_{0}(x) \mathrm{d} x=0$. Furthermore, let $Z_{n, 1}, \ldots, Z_{n, n}, n \in \mathbb{N}$, be a triangular array of rowwise i.i.d. random variables with Lebesgue density

$$
f_{n}(x)=f_{0}(x)\left(1+\frac{c(x)}{\sqrt{n}}\right), \quad x>0,
$$


where we assume $n$ to be large enough to ensure the non-negativity of $f_{n}$. The $n$-fold product measure of $f_{n}(x) \mathrm{d} x$ is contiguous to the $n$-fold product measure of $f_{0}(x) \mathrm{d} x$ in terms of [35].

Theorem 3. Under the stated assumptions, we have

$$
T_{n}=T_{n}\left(Z_{n, 1}, \ldots, Z_{n, n}\right) \stackrel{\mathcal{D}}{\longrightarrow}\|\mathcal{W}+\zeta\|_{L^{2}}^{2}, \text { as } n \rightarrow \infty,
$$

where $\zeta(\cdot)=\int_{0}^{\infty} g_{\varphi}(\cdot, x) c(x) f_{0}(x) \mathrm{d} x$, and $\mathcal{W}$ is the centered Gaussian element of $L^{2}$ from Theorem 2 with the function $g_{\varphi}(\cdot, \cdot)$ related to the covariance kernel of $\mathcal{W}$ as in Theorem 2.

We thus conclude that our test has non-trivial power against contiguous alternatives of the considered kind.

In the next section we explain how the test is implemented using a parametric bootstrap procedure. We also show that Theorem 2 can be used to prove that the bootstrap is asymptotically valid. However, we would also like to show that the bootstrap-based test is consistent against fixed alternatives. To achieve this goal, we provide another limit result, this time considering the setting under alternative distributions. Let $X$ be any positive random variable with $\mathbb{E}[X]<\infty$ and $\mathbb{E}\left[X^{-1}\right]<\infty$ (and $\mathbb{E}\left[X^{2}\right]<\infty$ if moment estimators are used), and let $X_{1}, X_{2}, \ldots$ be i.i.d. copies of $X$. We assume that either the ML or the moment estimators are considered, and we thus have, from Remark 1 that

$$
\left(\widehat{\mu}_{n}, \widehat{\lambda}_{n}\right) \longrightarrow(\mu, \lambda)
$$

$\mathbb{P}$-a.s., as $n \rightarrow \infty$, for some $\mu, \lambda>0$ (where we can specify $\mu=\mathbb{E}[X]$ ). In view of the scale invariance of $T_{n}$, we assume $\mu=\mathbb{E}[X]=1$, and have $\widehat{\varphi}_{n}=\widehat{\lambda}_{n} / \widehat{\mu}_{n} \longrightarrow \lambda / \mu=$ $\lambda=: \varphi \mathbb{P}$-a.s., as $n \rightarrow \infty$, where $\varphi$ may be specified as in Remark 1. By straightforward approximations and standard Glivenko-Cantelli arguments similar to the proof of Theorem 5 of [26], the following functional law of large numbers is obtained.

Theorem 4. As $n \rightarrow \infty$, we have

$$
\frac{T_{n}}{n} \stackrel{\mathbb{P}}{\longrightarrow} \Delta_{\varphi}=\int_{0}^{\infty}\left|\mathbb{E}\left[\frac{1}{2}\left(\varphi+\frac{3}{X}-\frac{\varphi}{X^{2}}\right) \min \{X, t\}\right]-\mathbb{P}(X \leq t)\right|^{2} w(t) \mathrm{d} t .
$$

Because of Theorem 1 the limit in Theorem 4 is 0 if, and only if, $X$ follows some inverse Gaussian distribution $\operatorname{IG}(1, \varphi)$, and it is strictly greater than 0 otherwise. This result will prove the consistency of the procedure in the next section.

\section{The Bootstrap Procedure and a Consistency Result}

In practice, to carry out the test at some level $\alpha \in(0,1)$, we suggest a parametric bootstrap procedure, as the distribution of the test statistic depends on an unknown parameter and is very complicated. The approach is as follows. Given a sample $X_{1}, \ldots, X_{n}$ of i.i.d. positive random variables with $\mathbb{E}\left[X_{1}+X_{1}^{-1}\right]<\infty$ (and $\mathbb{E}\left[X_{1}^{2}\right]<\infty$ when moment estimation is used), calculate $T_{n}\left(X_{1}, \ldots, X_{n}\right)$, using for instance the explicit formulae from Section 5.1. Additionally we calculate the estimators $\widehat{\mu}_{n}\left(X_{1}, \ldots, X_{n}\right)$ and $\widehat{\lambda}_{n}\left(X_{1}, \ldots, X_{n}\right)$, and put $\widehat{\varphi}_{n}\left(X_{1}, \ldots, X_{n}\right)=\widehat{\lambda}_{n}\left(X_{1}, \ldots, X_{n}\right) / \widehat{\mu}_{n}\left(X_{1}, \ldots, X_{n}\right)$. Conditional on this value of $\widehat{\varphi}_{n}$, generate $b$ samples of size $n$ from the IG $\left(1, \widehat{\varphi}_{n}\right)$-distribution, and calculate the test statistic for each of them, with the parameters estimated from the bootstrap sample in every instance. This yields the values $T_{n, 1}^{*}, \ldots, T_{n, b}^{*}$. Define the empirical distribution function

$$
F_{n, b}^{*}(t)=\frac{1}{b} \sum_{k=1}^{b} \mathbb{1}\left\{T_{n, k}^{*} \leq t\right\}, \quad t>0,
$$


and the empirical $(1-\alpha)$-quantile, $c_{n, b}^{*}(\alpha)=F_{n, b}^{*-1}(1-\alpha)$, of $T_{n, 1}^{*}, \ldots, T_{n, b}^{*}$. The hypothesis (2) is rejected if $T_{n}\left(X_{1}, \ldots, X_{n}\right)>c_{n, b}^{*}(\alpha)$. Denote by $F_{\varphi}$ the distribution function of $\|\mathcal{W}\|_{L^{2}}^{2}$ under the $\operatorname{IG}(1, \varphi)$-distribution as in Theorem 2, where $\varphi$ is the (almost sure) limit of $\widehat{\varphi}_{n}$ which exists by Remark 1. It is straightforward to adapt the methods from [36], applying Theorem 2, to prove that

$$
\sup _{t>0}\left|F_{n, b}^{*}(t)-F_{\varphi}(t)\right| \stackrel{\mathbb{P}}{\longrightarrow} 0, \quad \text { as } n, b \rightarrow \infty,
$$

and thus $c_{n, b}^{*}(\alpha) \stackrel{\mathbb{P}}{\longrightarrow} F_{\varphi}^{-1}(1-\alpha) \in(0, \infty)$, as $n, b \rightarrow \infty$. Hence, if $X_{1} \sim \operatorname{IG}(\mu, \lambda)$ for some $\mu, \lambda>0$, then

$$
\mathbb{P}\left(T_{n}\left(X_{1}, \ldots, X_{n}\right)>c_{n, b}^{*}(\alpha)\right) \longrightarrow \alpha, \quad \text { as } n, b \rightarrow \infty .
$$

We conclude that a given level of significance is attained in the limit, as both the sample size and the bootstrap size approach infinity. The bootstrap procedure is thus asymptotically valid.

Now suppose that $X_{1}$ from above does not follow any inverse Gaussian law. Then, the limit $\Delta_{\varphi}$ figuring in Theorem 4 is strictly positive. Consequently, Theorem 4 and the results on the bootstrap critical values above imply

$$
\mathbb{P}\left(T_{n}\left(X_{1}, \ldots, X_{n}\right)>c_{n, b}^{*}(\alpha)\right) \longrightarrow 1, \quad \text { as } n, b \rightarrow \infty,
$$

i.e., our test is consistent (in the bootstrap setting) against any such alternative distribution. We suggest using the above bootstrap procedure when the test is applied in practice. In the extensive power approximation in the following section, the procedure becomes very demanding due to the high number of Monte Carlo runs. To accelerate the computations, we employ in that simulation study the so-called warp-speed bootstrap, see [37], as explained in Section 5.

\section{Monte Carlo Study and Real Data Examples}

This section compares the finite sample power performance of the newly proposed test to that of competing tests for the inverse Gaussian distribution. Below, we consider the computational form of our test statistic. Then, various existing goodness-of-fit tests for the inverse Gaussian distribution are discussed. Finally, the power calculations, including the considered alternative distributions and the warp-speed bootstrap methodology, are detailed. Sample sizes of 30 and 50 are considered throughout.

\subsection{Computational Form}

We start with computationally stable representations of the test statistic for the weight functions $w_{a}(t)=\exp (-a t)$ and $\widetilde{w}_{a}(t)=\exp \left(-a t^{2}\right), t>0$. For $w_{a}$ we define

$$
\begin{aligned}
& h_{1, a}(s, t)=\left\{\begin{array}{l}
\frac{1}{a^{3}}\left(2-e^{-a s}\left((a s+1)^{2}+1\right)\right)+\frac{s}{a^{2}}\left(e^{-a s}(a s+1)-e^{-a t}(a t+1)\right) \\
+\frac{s t}{a} e^{-a t}, \quad s \leq t, \\
\frac{1}{a^{3}}\left(2-e^{-a t}\left((a t+1)^{2}+1\right)\right)+\frac{t}{a^{2}}\left(e^{-a t}(a t+1)-e^{-a s}(a s+1)\right) \\
+\frac{s t}{a} e^{-a s}, \quad s>t,
\end{array}\right. \\
& h_{2, a}(s, t)=\left\{\begin{array}{l}
\frac{s}{a} e^{-a t}, \quad s \leq t, \\
\frac{1}{a^{2}}\left(e^{-a t}(a t+1)-e^{-a s}(a s+1)\right)+\frac{s}{a} e^{-a s}, \quad s>t,
\end{array}\right.
\end{aligned}
$$


and obtain a numerically stable version of the test statistic,

$$
\begin{aligned}
T_{n, a}=\frac{1}{4 n} \sum_{j, k=1}^{n} & \left(\widehat{\varphi}_{n}+\frac{3}{Y_{n, j}}-\frac{\widehat{\varphi}_{n}}{Y_{n, j}^{2}}\right)\left(\widehat{\varphi}_{n}+\frac{3}{Y_{n, k}}-\frac{\widehat{\varphi}_{n}}{Y_{n, k}^{2}}\right) h_{1, a}\left(Y_{n, j}, Y_{n, k}\right) \\
& -2\left(\widehat{\varphi}_{n}+\frac{3}{Y_{n, j}}-\frac{\widehat{\varphi}_{n}}{Y_{n, j}^{2}}\right) h_{2, a}\left(Y_{n, j}, Y_{n, k}\right) \\
& -2\left(\widehat{\varphi}_{n}+\frac{3}{Y_{n, k}}-\frac{\widehat{\varphi}_{n}}{Y_{n, k}^{2}}\right) h_{2, a}\left(Y_{n, k}, Y_{n, j}\right)+\frac{4}{a} e^{-a \max \left(Y_{n, j}, Y_{n, k}\right),}
\end{aligned}
$$

where $Y_{n, 1}, \ldots, Y_{n, n}$ and $\widehat{\varphi}_{n}$ are as in Section 1 . For the second weight, $\widetilde{w}_{a}$, define

$$
\begin{aligned}
& \widetilde{h}_{1, a}(s, t)=\left\{\begin{array}{l}
\frac{\sqrt{\pi / a^{3}}}{4}-\frac{a}{2} \sqrt{\frac{\pi}{a^{5}}} \Phi(-\sqrt{2 a} s)-\frac{s}{2 a} e^{-a t^{2}}+s t \sqrt{\frac{\pi}{a}} \Phi(-\sqrt{2 a} t), \quad s \leq t, \\
\frac{\sqrt{\pi / a^{3}}}{4}-\frac{a}{2} \sqrt{\frac{\pi}{a^{5}}} \Phi(-\sqrt{2 a} t)-\frac{t}{2 a} e^{-a s^{2}}+s t \sqrt{\frac{\pi}{a}} \Phi(-\sqrt{2 a} s), \quad s>t,
\end{array}\right. \\
& \widetilde{h}_{2, a}(s, t)=\left\{\begin{array}{l}
s \sqrt{\frac{\pi}{a}} \Phi(-\sqrt{2 a} t), \quad s \leq t, \\
\frac{1}{2 a}\left(e^{-a t^{2}}-e^{-a s^{2}}\right)+s \sqrt{\frac{\pi}{a}} \Phi(-\sqrt{2 a} s), \quad s>t,
\end{array}\right.
\end{aligned}
$$

where $\Phi$ denotes the distribution function of the standard normal distribution. Then we have a numerically stable version of the corresponding test statistic, namely

$$
\begin{aligned}
\widetilde{T}_{n, a}=\frac{1}{4 n} \sum_{j, k=1}^{n}( & \left.\widehat{\varphi}_{n}+\frac{3}{Y_{n, j}}-\frac{\widehat{\varphi}_{n}}{Y_{n, j}^{2}}\right)\left(\widehat{\varphi}_{n}+\frac{3}{Y_{n, k}}-\frac{\widehat{\varphi}_{n}}{Y_{n, k}^{2}}\right) \widetilde{h}_{1, a}\left(Y_{n, j}, Y_{n, k}\right) \\
& -2\left(\widehat{\varphi}_{n}+\frac{3}{Y_{n, j}}-\frac{\widehat{\varphi}_{n}}{Y_{n, j}^{2}}\right) \widetilde{h}_{2, a}\left(Y_{n, j}, Y_{n, k}\right) \\
& -2\left(\widehat{\varphi}_{n}+\frac{3}{Y_{n, k}}-\frac{\widehat{\varphi}_{n}}{Y_{n, k}^{2}}\right) \widetilde{h}_{2, a}\left(Y_{n, k}, Y_{n, j}\right) \\
+ & 4 \sqrt{\frac{\pi}{a}} \Phi\left(-\sqrt{2 a} \max \left(Y_{n, j}, Y_{n, k}\right)\right) .
\end{aligned}
$$

\subsection{Existing Tests of Fit for the Inverse Gaussian Distribution}

The set of competing tests we consider comprises several classical tests as well as more recent tests. We choose the following procedures:

1. The Kolmogorov-Smirnov test,

3. the Anderson-Darling test,

5. a test from [16],
2. the Cramér-von Mises test,

4. two tests proposed in [13],

6. a test from [8].

Below, we briefly provide the details of these tests. The first three tests are well known, and we only provide the computational form of the test statistic in each case. The remaining four tests are considered in more detail. The tests by $[8,16]$ are quite recent, while the tests by [13] are included due to their impressive power performance in previous empirical studies. For a recent literature overview concerning the existing tests for the inverse Gaussian distribution, see [14].

\subsubsection{Classical Tests}

Let $X_{(j)}$ denote the $j$ th order statistic of $X_{1}, \ldots, X_{n}$. Furthermore, let $\widehat{F}(x)=F\left(x ; \widehat{\mu}_{n}, \widehat{\lambda}_{n}\right)$, where $F$ is the distribution function of the inverse Gaussian distribution. For each of the following tests, the null hypothesis is rejected for large values of the test statistic. 
1. Kolmogorov-Smirnov test: The form of the test statistic is

$$
K S=\max \left(D^{+}, D^{-}\right),
$$

where $D^{+}=\max _{j=1, \ldots, n}\left(\frac{j}{n}-\widehat{F}\left(X_{(j)}\right)\right)$ and $D^{-}=\max _{j=1, \ldots, n}\left(\widehat{F}\left(X_{(j)}\right)-\frac{j-1}{n}\right)$.

2. Cramér-von Mises test: In this case, the test statistic is

$$
C M=\frac{1}{12 n}+\sum_{j=1}^{n}\left(\widehat{F}\left(X_{(j)}\right)-\frac{2 j-1}{2 n}\right)^{2}
$$

3. Anderson-Darling test: The computational form of the Anderson-Darling test statistic is

$$
A D=-n-\frac{1}{n} \sum_{j=1}^{n}\left[(2 j-1) \log \widehat{F}\left(X_{(j)}\right)+(2(n-j)+1) \log \left(1-\widehat{F}\left(X_{(j)}\right)\right)\right]
$$

\subsubsection{Tests Proposed by Henze and Klar}

In [13] two classes of tests for the inverse Gaussian distribution based on the empirical Laplace transform are proposed. The Laplace transform of the $\operatorname{IG}(\mu, \lambda)$ distribution is given by $L(t)=\mathbb{E}[\exp (-t X)]=\exp \left(\frac{\lambda}{\mu}\left(1-\sqrt{1+\frac{2 \mu^{2} t}{\lambda}}\right)\right), t \geq 0$. As a result, the Laplace transform of the inverse Gaussian distribution satisfies the characteristic differential equation

$$
\mu L(t)+\sqrt{1+\frac{2 \mu^{2} t}{\lambda}} L^{\prime}(t)=0, \quad t \geq 0,
$$

subject to the initial condition $L(0)=1$. The empirical Laplace transform is $L_{n}(t)=$ $n^{-1} \sum_{j=1}^{n} \exp \left(-t X_{j}\right)$. Under the assumption that $X_{1}, \ldots, X_{n}$ are realized from an inverse Gaussian distribution, (6) suggests that $\widetilde{\varepsilon}_{n}(t)=\widehat{\mu}_{n} L_{n}(t)+L_{n}^{\prime}(t) \sqrt{1+2 t \widehat{\mu}_{n}^{2} / \widehat{\lambda}_{n}}$ is close to zero for each value of $t$. The proposed class of test statistics thus is

$$
H K_{n, a}^{(1)}=\frac{n}{\widehat{\mu}_{n}} \int_{0}^{\infty}\left(\widetilde{\varepsilon}_{n}(t)\right)^{2} \exp \left(-a \widehat{\mu}_{n} t\right) \mathrm{d} t,
$$

where $\widehat{\mu}_{n}$ and $\widehat{\lambda}_{n}$ denote the maximum likelihood estimates of $\mu$ and $\lambda$, respectively, and $a \geq 0$ is a tuning parameter. Due to the intractability of some of the calculations required for the implementation of the test statistic, the authors recommend the use of the exponentially scaled complementary error function erfce $(x)=2 \exp \left(x^{2}\right) \pi^{-1} \int_{x}^{\infty} \exp \left(-t^{2}\right) \mathrm{d} t$ rather than the distribution function of a Gaussian random variable in the value of the test statistic. Note that $\operatorname{erfce}(x)$ tends to $\infty$ for small values of $x$. Furthermore, for sufficiently large values of $x$, the numerical evaluation of erfce $(x)$ breaks down, since the value of the integral and the exponential function are calculated to be 0 and $\infty$, respectively, by all standard statistical software packages. The latter difficulty can be overcome by noting that $\lim _{x \rightarrow \infty} \operatorname{erfce}(x)=0$. As a result, the use of the erfce function reduces the numerical problems encountered when implementing the tests proposed in [13] without removing these difficulties altogether.

Letting $\widehat{\varphi}_{n}=\widehat{\lambda}_{n} / \widehat{\mu}_{n}$ and $Y_{n, j}=X_{j} / \widehat{\mu}_{n}$ as in Section 1 , and $\widehat{Z}_{j k}=\widehat{\varphi}_{n} \cdot\left(Y_{n, j}+Y_{n, k}+a\right)$, the test statistic can be expressed in a tractable form, namely

$$
\begin{aligned}
& H K_{n, a}^{(1)}=\frac{\widehat{\varphi}_{n}}{n} \sum_{j, k=1}^{n} \widehat{Z}_{j k}^{-1} . \\
& \left\{1-\left(Y_{n, j}+Y_{n, k}\right)\left(1+\sqrt{\frac{\pi}{2 \widehat{Z}_{j k}}} \operatorname{erfce}\left(\sqrt{\frac{\widehat{Z}_{j k}}{2}}\right)\right)+\left(1+\frac{2}{\widehat{Z}_{j k}}\right) Y_{n, j} Y_{n, k}\right\} .
\end{aligned}
$$


The null hypothesis is rejected for large values of $H K_{n, a}^{(1)}$. Based on the power performance of this class of tests, the authors recommend the use of $a=0$. This recommendation is met to obtain the numerical results shown below.

Henze and Klar [13] also proposed a second, more immediate class of tests based on the empirical Laplace transform. This second class of tests is based on the difference between the Laplace transform of the $\operatorname{IG}\left(\widehat{\mu}_{n}, \widehat{\lambda}_{n}\right)$ distribution (denoted by $\widehat{L}$ ) and the empirical Laplace transform:

$$
H K_{n, a}^{(2)}=n \widehat{\mu}_{n} \int_{0}^{\infty}\left(L_{n}(t)-\widehat{L}(t)\right)^{2} \exp \left(-a \widehat{\mu}_{n} t\right) \mathrm{d} t
$$

for some $a \geq 0$. Two distinct computational forms for the test statistic are obtained, distinguishing the cases $a=0$ and $a>0$. Again, the recommended value of the tuning parameter is $a=0$. In this case, the test statistic can be expressed as

$$
\begin{aligned}
H K_{n, 0}^{(2)} & =\frac{1}{n} \sum_{j, k=1}^{n} Z_{j k}^{-1}-2 \sum_{j=1}^{n} Y_{n, j}^{-1}\left\{1-\sqrt{\frac{\pi \widehat{\varphi}_{n}}{2 Y_{n, j}}} \operatorname{erfce}\left(\frac{\widehat{\varphi}_{n}^{1 / 2}\left(Y_{n, j}+1\right)}{\sqrt{2 Y_{n, j}}}\right)\right\} \\
& +n \frac{1+2 \widehat{\varphi}_{n}}{4 \widehat{\varphi}_{n}},
\end{aligned}
$$

where $Z_{j k}=Y_{n, j}+Y_{n, k}$. The hypothesis that the data are realized from an inverse Gaussian distribution is rejected for large values of the test statistic.

\subsubsection{The Test of Villaseñor and Gonzáles-Estrada}

In [16] three goodness-of-fit tests for the inverse Gaussian distribution are introduced, the most powerful of which is discussed below. Using the notation from the mentioned paper, it can be shown that if $X \sim \operatorname{IG}(\mu, \lambda)$, then $Z^{(\mu)}=(X-\mu)^{2} / X$ follows a Gamma distribution with shape parameter $1 / 2$ and scale parameter $2 \mu^{2} / \lambda$. A goodness-of-fit test for the inverse Gaussian distribution can be constructed using the moment estimator of $\operatorname{Cov}\left(X, Z^{(\mu)}\right)$, where $\mu$ is estimated by $\widehat{\mu}_{n}=\bar{X}_{n}$. The suggested test statistic can be written as

$$
V G_{n}=\sqrt{\frac{n \widehat{\lambda}_{n}}{6 \widehat{\mu}_{n}}}\left(\frac{\widehat{\lambda}_{n} S_{n}^{2}}{\widehat{\mu}_{n}^{3}}-1\right)
$$

where $S_{n}^{2}$ denotes the sample variance of $X_{1}, \ldots, X_{n}$, and where $\widehat{\lambda}_{n}$ is the ML estimator of $\lambda$. The asymptotic distribution of $V G_{n}$ is standard normal, and the null hypothesis is rejected for large values of $\left|V G_{n}\right|$.

\subsubsection{The Test of Baringhaus and Gaigall}

The following characterization of the class of inverse Gaussian distributions is provided by [38]. Let $X_{1}, X_{2}$ be positive, identically distributed random variables with finite moments $\mathbb{E}\left[X_{1}^{2}\right]$ and $\mathbb{E}\left[X_{1}^{-1}\right]$. In this case, $Y=\left(X_{1}+X_{2}\right) / 2$ and $Z=\left(X_{1}^{-1}+X_{2}^{-1}\right) / 2-Y^{-1}$ are independent if, and only if, $X_{1}$ and $X_{2}$ are inverse Gaussian. This result is the basis of the test in [8] (where similar tests for various other distributions are also constructed) as follows. Let $f\left(X_{1}, X_{2}\right)$ and $g\left(X_{1}, X_{2}\right)$ respectively denote the transformations used to obtain $Y$ and $Z$ above. Consider the bivariate random vectors $\left(\widetilde{Y}_{i, j}, \widetilde{Z}_{i, j}\right)=\left(f\left(X_{i}, X_{j}\right), g\left(X_{i}, X_{j}\right)\right)$, $1 \leq i, j \leq n, i \neq j$, the coordinates of which are independent if, and only if, $X_{1}, \ldots, X_{n}$ are realized from an inverse Gaussian distribution. The novel testing procedure proposed by [8] entails using the Hoeffding-Blum-Kiefer-Rosenblatt independence criterion. They arrive at the test statistic

$$
B G_{n}=\frac{n}{(n(n-1))^{5}} \sum_{\mu, v=1, \mu \neq v}^{n}\left(N_{1}(\mu, v) N_{4}(\mu, v)-N_{2}(\mu, v) N_{3}(\mu, v)\right)^{2},
$$


where

$$
\begin{aligned}
& N_{1}(\mu, v)=\sum_{i, j=1, i \neq j}^{n} \mathbb{1}\left\{\widetilde{Y}_{i, j} \leq \widetilde{Y}_{\mu, v}, \widetilde{Z}_{i, j} \leq \widetilde{Z}_{\mu, v}\right\}, \\
& N_{2}(\mu, v)=\sum_{i, j=1, i \neq j}^{n} \mathbb{1}\left\{\widetilde{Y}_{i, j} \leq \widetilde{Y}_{\mu, v}, \widetilde{Z}_{i, j}>\widetilde{Z}_{\mu, v}\right\}, \\
& N_{3}(\mu, v)=\sum_{i, j=1, i \neq j}^{n} \mathbb{1}\left\{\widetilde{Y}_{i, j}>\widetilde{Y}_{\mu, v}, \widetilde{Z}_{i, j} \leq \widetilde{Z}_{\mu, v}\right\}, \\
& N_{4}(\mu, v)=\sum_{i, j=1, i \neq j}^{n} \mathbb{1}\left\{\widetilde{Y}_{i, j}>\widetilde{Y}_{\mu, v}, \widetilde{Z}_{i, j}>\widetilde{Z}_{\mu, v}\right\} .
\end{aligned}
$$

The hypothesis that the data are realized from an inverse Gaussian distribution is rejected for large values of $B G_{n}$.

\subsection{Power Calculations}

Table 1 shows the alternative distributions considered in the empirical study below. Several of the listed distributions are investigated for various parameter values. The powers of the tests against the inverse Gaussian distribution with mean parameter 1 and shape parameter $\theta$, denoted in Tables 2 and 3 by $\operatorname{IG}(\theta)$, are also calculated for several values of $\theta$ to evaluate the empirical size of all competing tests. For the choices of the parameters, we very roughly align with the study by [13].

Since the null distribution of the test statistic depends on the unknown value of the shape parameter, we use a parametric bootstrap, as explained in Section 4, to calculate critical values for the test statistics under consideration. Given that the parametric bootstrap is computationally demanding, we employ the warp-speed method proposed by [37] to approximate the power of all the considered tests. We denote the number of Monte Carlo replications by $M C$, and recall that this method capitalizes on the repetition inherent in the Monte Carlo simulation to produce bootstrap replications, rather than relying on a separate 'bootstrap loop'. The procedure can be summarized as follows.

1. Obtain a sample $X_{1}, \ldots, X_{n}$ from a distribution, say $F$, and estimate $\mu$ and $\lambda$ by $\widehat{\mu}_{n}$ and $\widehat{\lambda}_{n}$, respectively. Additionally, let $\widehat{\varphi}_{n}=\widehat{\lambda}_{n} / \widehat{\mu}_{n}$.

2. Obtain the scaled data $Y_{n, j}=X_{j} / \widehat{\mu}_{n}$ for each $j=1, \ldots, n$, and calculate the test statistic, $S=S\left(Y_{n, 1}, \ldots, Y_{n, n}\right)$, say.

3. Generate a bootstrap sample $X_{1}^{*}, \ldots, X_{n}^{*}$ by independently sampling from $\operatorname{IG}\left(1, \widehat{\varphi}_{n}\right)$. Then proceed to calculate $\widehat{\mu}_{n}^{*}=\widehat{\mu}_{n}\left(X_{1}^{*}, \ldots, X_{n}^{*}\right)$ and $\widehat{\lambda}_{n}^{*}=\widehat{\lambda}_{n}\left(X_{1}^{*}, \ldots, X_{n}^{*}\right)$.

4. Scale the values in the bootstrap sample using $Y_{n, j}^{*}=X_{j}^{*} / \widehat{\mu}_{n}^{*}, j=1, \ldots, n$, and determine the value of the test statistic for these scaled bootstrap values, i.e., $S^{*}=$ $S\left(Y_{n, 1}^{*}, \ldots, Y_{n, n}^{*}\right)$.

5. Repeat steps 1 to $4 M C$ times to obtain $S_{1}, \ldots, S_{M C}$ and $S_{1}^{*}, \ldots, S_{M C}^{*}$, where $S_{m}$ denotes the value of the test statistic calculated from the $m$ th scaled sample data generated in step 1 , and $S_{m}^{*}$ denotes the value of the bootstrap test statistic calculated from the single scaled bootstrap sample obtained in the $m$ th iteration of the Monte Carlo simulation.

6. To obtain the power approximation, reject the null hypothesis for the $m$ th sample whenever $S_{m}>S_{(\lfloor M C \cdot(1-\alpha)\rfloor)}^{*} m=1, \ldots, M C$, where $S_{(1)}^{*} \leq \ldots \leq S_{(M C)}^{*}$ are the ordered values of the statistics obtained from the bootstrap samples and $\lfloor\cdot\rfloor$ denotes the floor function. 
Table 1. Alternative distributions considered.

\begin{tabular}{clc}
\hline Alternative & Density & Notation \\
\hline Weibull & $\theta x^{\theta-1} \exp \left(-x^{\theta}\right)$ & $W(\theta)$ \\
Lognormal & $\exp \left\{-\frac{1}{2}\left(\frac{\log (x)}{\theta}\right)^{2}\right\} /\{\theta x \sqrt{2 \pi}\}$ & $L N(\theta)$ \\
Gamma & $\frac{1}{\Gamma(\theta)} x^{\theta-1} \exp (-x)$ & $\Gamma(\theta)$ \\
Dhillon & $\frac{\theta+1}{x+1} \exp \left\{-(\log (x+1))^{\theta+1}\right\}(\log (x+1))^{\theta}$ & $D H(\theta)$ \\
Uniform & $\frac{1}{\theta_{2}-\theta_{1}} \cdot \mathbb{1}\left\{\theta_{1} \leq x \leq \theta_{2}\right\}$ & $U\left(\theta_{1}, \theta_{2}\right)$ \\
Half Cauchy & $2\left[\pi \theta\left(1+\left(\frac{x}{\theta}\right)^{2}\right)\right]^{-1}, x>0$ & $H C(\theta)$
\end{tabular}

The warp-speed methodology described above is a computationally efficient alternative to the classical parametric bootstrap procedure (with a separate 'bootstrap loop') usually employed in power calculations in the presence of a shape parameter. The latter method is detailed and implemented in Section 5 of [13]. Extensive Monte Carlo simulations indicate that the power estimates obtained using the two bootstrap methods provide almost identical results for all but two of the test statistics used. The tests for which the power estimates differ are those proposed in [13]. For these tests, the classical parametric bootstrap approach typically provides power estimates that are higher than those obtained using the warp-speed methodology. For the sake of consistency, the warp-speed methodology is used for all tests throughout the simulation study.

Tables 2 and 3 show the estimated powers obtained using the warp-speed bootstrap methodology with 50,000 replications for sample sizes 30 and 50 . The entries show the percentage of samples for which the null hypothesis was rejected, rounded to the closest integer. Following [13], the nominal significance level $\alpha$ is set to $10 \%$ throughout. To ease comparison, the highest power against each alternative distribution is printed in bold in the tables. We divide our new tests into four categories, depending on the weight function and estimation technique. More precisely, we implement the two weight functions $w_{a}$ and $\widetilde{w}_{a}$ as in Sections 1 and 5.1, distinguishing the resulting tests by the ' ' '-notation, and use upper indices $M L$ and $M O$ to indicate the use of maximum likelihood or moment estimators, respectively. For each of the resulting four classes, we consider three different values of the tuning parameter, namely $a=0.1,1,10$. Several other values were also investigated, but due to the remarkable insensitivity of the tests regarding the tuning parameter in most cases, we present only these three values in the numerical results. The mentioned insensitivity is particularly noticeable for the tests that employ the moment estimators.

For comparing the power results in Tables 2 and 3, some remarks are in order. Notice that each of the tests keeps the nominal level of $10 \%$ closely when the null hypothesis is true. Observing the powers associated with the existing tests for the inverse Gaussian distribution, it is clear that the power of the test by [16] is generally lower than the power of the remaining tests. Among the three classical procedures, the Anderson-Darling test performs best, while the $H K_{n, 0}^{(2)}$ test proposed by [13] produces the highest power among the more recent tests. 
Table 2. Approximated powers for sample size $n=30$.

\begin{tabular}{|c|c|c|c|c|c|c|c|c|c|c|c|c|c|c|c|c|c|c|c|}
\hline Distribution & $K S$ & $C M$ & $A D$ & $H K_{n, 0}^{(1)}$ & $H K_{n, 0}^{(2)}$ & $V G_{n}$ & $B G_{n}$ & $T_{n, 0.1}^{M L}$ & $T_{n, 1}^{M L}$ & $T_{n, 10}^{M L}$ & $T_{n, 0.1}^{M O}$ & $T_{n, 1}^{M O}$ & $T_{n, 10}^{M O}$ & $\widetilde{T}_{n, 0.1}^{M L}$ & $\widetilde{T}_{n, 1}^{M L}$ & $\widetilde{T}_{n, 10}^{M L}$ & $\widetilde{T}_{n, 0.1}^{M O}$ & $\widetilde{T}_{n, 1}^{M O}$ & $\widetilde{T}_{n, 10}^{M O}$ \\
\hline$I G(1)$ & 10 & 10 & 10 & 10 & 10 & 10 & 10 & 10 & 10 & 10 & 12 & 12 & 13 & 10 & 10 & 11 & 12 & 12 & 12 \\
\hline$I G(5)$ & 10 & 10 & 10 & 10 & 10 & 10 & 10 & 11 & 10 & 10 & 11 & 11 & 11 & 11 & 10 & 10 & 11 & 11 & 11 \\
\hline$I G(10)$ & 10 & 10 & 10 & 10 & 10 & 10 & 10 & 10 & 11 & 10 & 11 & 11 & 11 & 10 & 10 & 10 & 10 & 11 & 11 \\
\hline$I G(20)$ & 10 & 10 & 10 & 10 & 10 & 11 & 10 & 10 & 10 & 11 & 10 & 10 & 10 & 11 & 11 & 11 & 10 & 11 & 10 \\
\hline$W(1)$ & 80 & 83 & 84 & 61 & 68 & 30 & 43 & 82 & 86 & 85 & 94 & 93 & 95 & 85 & 84 & 85 & 94 & 93 & 94 \\
\hline$W(1.2)$ & 73 & 76 & 77 & 58 & 64 & 36 & 48 & 77 & 80 & 78 & 91 & 90 & 92 & 79 & 79 & 79 & 90 & 90 & 91 \\
\hline$W(1.6)$ & 62 & 67 & 69 & 54 & 58 & 42 & 55 & 69 & 73 & 69 & 86 & 85 & 87 & 71 & 72 & 70 & 86 & 85 & 86 \\
\hline$W(2)$ & 55 & 61 & 63 & 52 & 54 & 44 & 61 & 65 & 67 & 64 & 82 & 82 & 83 & 66 & 67 & 66 & 82 & 82 & 83 \\
\hline $\operatorname{LN}(0.6)$ & 14 & 15 & 16 & 17 & 18 & 14 & 17 & 15 & 16 & 20 & 20 & 19 & 22 & 15 & 16 & 19 & 19 & 19 & 21 \\
\hline$L N(1)$ & 21 & 22 & 23 & 22 & 25 & 15 & 14 & 17 & 20 & 28 & 27 & 26 & 29 & 18 & 20 & 24 & 26 & 25 & 27 \\
\hline $\operatorname{LN}(1.4)$ & 28 & 32 & 32 & 28 & 33 & 16 & 13 & 21 & 25 & 36 & 35 & 35 & 38 & 22 & 25 & 31 & 35 & 34 & 35 \\
\hline $\operatorname{LN}(2)$ & 42 & 46 & 46 & 37 & 44 & 17 & 24 & 26 & 34 & 48 & 49 & 48 & 52 & 28 & 33 & 41 & 49 & 48 & 49 \\
\hline $\operatorname{LN}(3)$ & 63 & 67 & 67 & 50 & 61 & 17 & 39 & 33 & 44 & 65 & 71 & 70 & 73 & 36 & 44 & 54 & 71 & 69 & 71 \\
\hline$\Gamma(1.5)$ & 61 & 65 & 66 & 53 & 57 & 36 & 47 & 63 & 68 & 68 & 83 & 82 & 85 & 66 & 67 & 68 & 83 & 82 & 83 \\
\hline$\Gamma(2)$ & 47 & 52 & 53 & 47 & 50 & 35 & 37 & 51 & 55 & 57 & 73 & 71 & 75 & 53 & 55 & 57 & 72 & 71 & 74 \\
\hline$\Gamma(2.5)$ & 39 & 42 & 44 & 41 & 44 & 31 & 30 & 43 & 47 & 50 & 65 & 64 & 67 & 44 & 47 & 50 & 64 & 63 & 66 \\
\hline$\Gamma(5)$ & 21 & 23 & 24 & 27 & 28 & 21 & 20 & 26 & 27 & 32 & 41 & 40 & 43 & 26 & 29 & 32 & 40 & 39 & 42 \\
\hline $\mathrm{DH}(1.5)$ & 35 & 39 & 39 & 38 & 41 & 29 & 26 & 37 & 42 & 47 & 57 & 57 & 61 & 39 & 42 & 46 & 57 & 56 & 58 \\
\hline$D H(2)$ & 33 & 37 & 38 & 38 & 40 & 29 & 28 & 39 & 42 & 45 & 58 & 57 & 60 & 39 & 42 & 46 & 57 & 56 & 59 \\
\hline$U(0.5,1.5)$ & 40 & 53 & 60 & 17 & 14 & 12 & 61 & 53 & 49 & 11 & 48 & 54 & 40 & 53 & 45 & 13 & 53 & 54 & 41 \\
\hline$U(1,2)$ & 34 & 46 & 54 & 6 & 5 & 1 & 48 & 35 & 34 & 4 & 19 & 25 & 14 & 36 & 31 & 4 & 26 & 26 & 14 \\
\hline$H C(1)$ & 61 & 64 & 63 & 44 & 53 & 16 & 30 & 32 & 47 & 64 & 44 & 43 & 46 & 35 & 48 & 58 & 44 & 42 & 43 \\
\hline
\end{tabular}


Table 3. Approximated powers for sample size $n=50$.

\begin{tabular}{|c|c|c|c|c|c|c|c|c|c|c|c|c|c|c|c|c|c|c|c|}
\hline Distribution & $K S$ & $C M$ & $A D$ & $H K_{n, 0}^{(1)}$ & $H K_{n, 0}^{(2)}$ & $V G_{n}$ & $B G_{n}$ & $T_{n, 0.1}^{M L}$ & $T_{n, 1}^{M L}$ & $T_{n, 10}^{M L}$ & $T_{n, 0.1}^{M O}$ & $T_{n, 1}^{M O}$ & $T_{n, 10}^{M O}$ & $\widetilde{T}_{n, 0.1}^{M L}$ & $\widetilde{T}_{n, 1}^{M L}$ & $\widetilde{T}_{n, 10}^{M L}$ & $\widetilde{T}_{n, 0.1}^{M O}$ & $\widetilde{T}_{n, 1}^{M O}$ & $\widetilde{T}_{n, 10}^{M O}$ \\
\hline$I G(1)$ & 10 & 10 & 10 & 10 & 10 & 10 & 10 & 10 & 10 & 11 & 11 & 11 & 11 & 10 & 10 & 10 & 11 & 11 & 11 \\
\hline$I G(5)$ & 10 & 10 & 10 & 10 & 10 & 10 & 10 & 10 & 10 & 10 & 11 & 10 & 11 & 11 & 10 & 10 & 10 & 10 & 10 \\
\hline$I G(10)$ & 10 & 10 & 10 & 10 & 10 & 10 & 10 & 10 & 10 & 10 & 11 & 10 & 10 & 11 & 10 & 10 & 10 & 10 & 10 \\
\hline$I G(20)$ & 10 & 10 & 10 & 10 & 10 & 10 & 10 & 10 & 10 & 10 & 11 & 10 & 10 & 11 & 10 & 10 & 10 & 10 & 10 \\
\hline$W(1)$ & 94 & 96 & 96 & 79 & 85 & 36 & 88 & 96 & 97 & 96 & 98 & 98 & 99 & 97 & 96 & 96 & 98 & 98 & 99 \\
\hline$W(1.2)$ & 89 & 92 & 92 & 77 & 82 & 45 & 83 & 93 & 95 & 92 & 98 & 97 & 98 & 94 & 94 & 93 & 97 & 97 & 98 \\
\hline$W(1.6)$ & 81 & 85 & 86 & 74 & 77 & 57 & 77 & 88 & 90 & 86 & 96 & 95 & 96 & 89 & 89 & 87 & 96 & 95 & 96 \\
\hline$W(2)$ & 74 & 80 & 82 & 71 & 74 & 62 & 74 & 85 & 86 & 82 & 94 & 94 & 94 & 86 & 86 & 84 & 94 & 94 & 94 \\
\hline$L N(0.6)$ & 16 & 18 & 18 & 20 & 21 & 15 & 12 & 17 & 18 & 23 & 21 & 20 & 23 & 17 & 18 & 22 & 20 & 20 & 21 \\
\hline$L N(1)$ & 26 & 29 & 30 & 29 & 33 & 18 & 17 & 21 & 26 & 35 & 28 & 27 & 31 & 22 & 26 & 31 & 28 & 27 & 28 \\
\hline$L N(1.4)$ & 38 & 42 & 43 & 39 & 44 & 19 & 24 & 27 & 35 & 47 & 36 & 35 & 40 & 29 & 36 & 41 & 36 & 34 & 36 \\
\hline$L N(2)$ & 57 & 62 & 62 & 52 & 60 & 18 & 37 & 36 & 49 & 63 & 49 & 48 & 53 & 41 & 49 & 56 & 49 & 48 & 50 \\
\hline $\operatorname{LN}(3)$ & 81 & 84 & 84 & 68 & 78 & 18 & 60 & 48 & 66 & 82 & 72 & 71 & 75 & 56 & 65 & 74 & 71 & 71 & 72 \\
\hline$\Gamma(1.5)$ & 79 & 83 & 84 & 72 & 76 & 48 & 70 & 83 & 87 & 85 & 93 & 93 & 94 & 86 & 85 & 86 & 93 & 92 & 94 \\
\hline$\Gamma(2)$ & 65 & 71 & 72 & 64 & 68 & 48 & 55 & 70 & 75 & 75 & 85 & 85 & 88 & 73 & 74 & 75 & 85 & 84 & 87 \\
\hline$\Gamma(2.5)$ & 53 & 59 & 60 & 58 & 60 & 45 & 45 & 59 & 64 & 67 & 77 & 76 & 80 & 61 & 64 & 67 & 77 & 76 & 79 \\
\hline$\Gamma(5)$ & 27 & 31 & 32 & 38 & 39 & 30 & 27 & 36 & 39 & 43 & 51 & 50 & 54 & 36 & 40 & 44 & 50 & 49 & 53 \\
\hline$D H(1.5)$ & 48 & 53 & 54 & 53 & 56 & 38 & 39 & 52 & 58 & 63 & 69 & 67 & 73 & 55 & 58 & 61 & 68 & 67 & 71 \\
\hline$D H(2)$ & 46 & 51 & 53 & 53 & 56 & 42 & 41 & 54 & 58 & 61 & 71 & 70 & 74 & 56 & 58 & 61 & 71 & 69 & 73 \\
\hline$U(0.5,1.5)$ & 61 & 76 & 86 & 29 & 24 & 30 & 84 & 80 & 76 & 15 & 69 & 76 & 56 & 80 & 72 & 18 & 75 & 76 & 58 \\
\hline$U(1,2)$ & 51 & 69 & 80 & 8 & 7 & 5 & 71 & 63 & 60 & 5 & 30 & 41 & 19 & 62 & 56 & 4 & 40 & 40 & 19 \\
\hline$H C(1)$ & 77 & 80 & 80 & 58 & 68 & 15 & 49 & 46 & 68 & 81 & 39 & 38 & 40 & 53 & 69 & 77 & 39 & 38 & 39 \\
\hline
\end{tabular}


Turning our attention to a global comparison between the tests, we see that our newly proposed method outperforms the existing tests in most cases considered. In fact, the new tests produce higher powers in 17 out of 20 alternative distributions for both sample sizes. Interestingly, the tests using the moment estimators outperform those based on the maximum likelihood estimates. Although the choice of the weight function seems to have a smaller influence on the power than the estimation method, the test statistics which employ $w_{a}(t)=\exp (-a t)$ as a weight outperform those that use $\widetilde{w}_{a}(t)=\exp \left(-a t^{2}\right)$. Based on the numerical results, we recommend for practical applications the statistic $T_{n, 10}^{M O}$.

Figure 1 represents a snapshot of the empirical powers for various sample sizes for 5 fairly powerful tests against the Gamma and Weibull alternatives, both with parameter 2 . These powers are obtained by the warp-speed bootstrap methodology with 50,000 replications for sample sizes $n=\{10,20,30, \ldots, 100\}$. Again, one can see that $T_{n, 10}^{M O}$ outperforms the other 4 tests for all sample sizes considered.
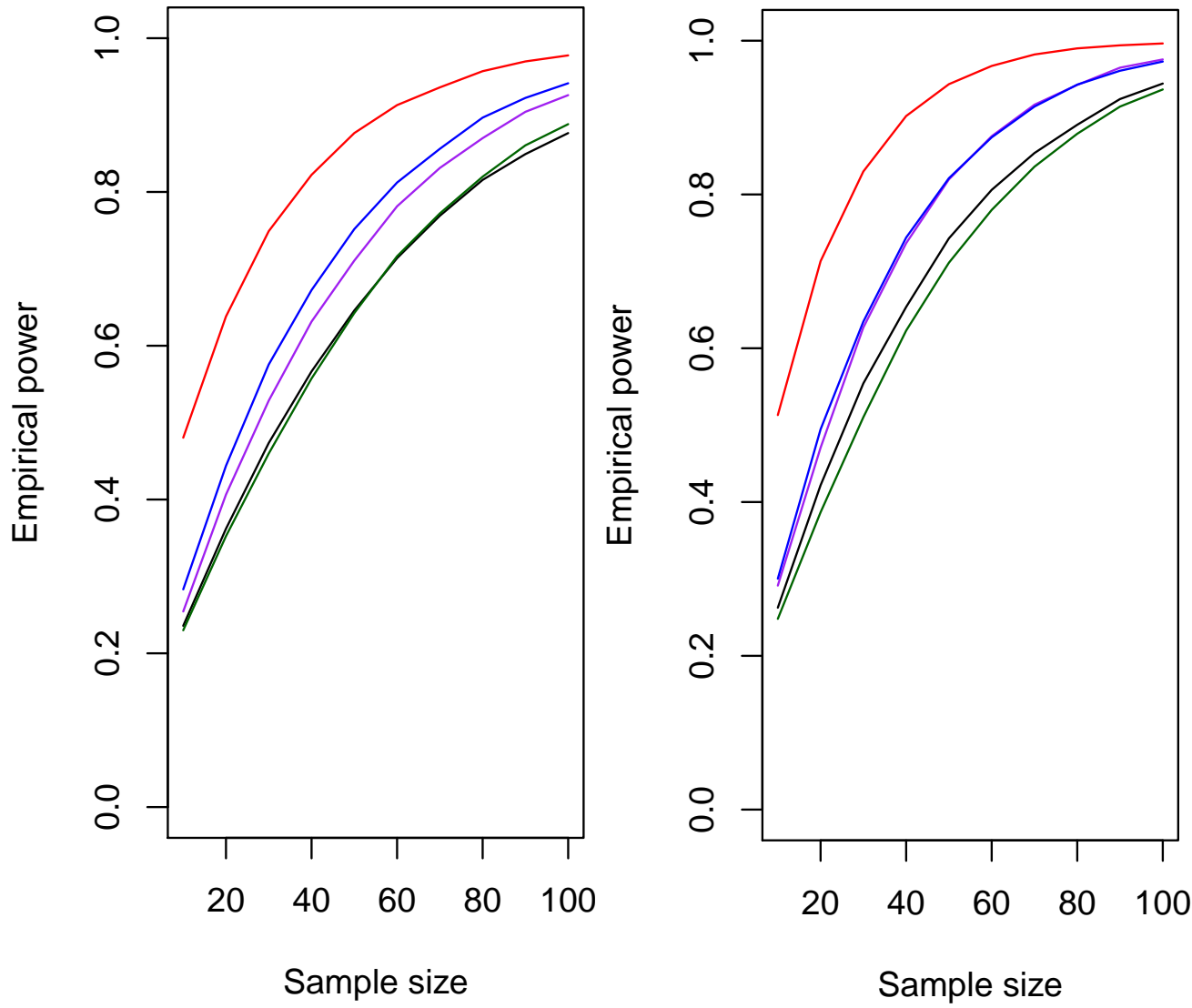

Figure 1. Empirical powers for various sample sizes against the $\Gamma(2)$ (left) and the $W(2)$ (right) alternatives. The tests considered are $K S$ (black line), $A D$ (purple line), $H K_{n, 0}^{(2)}$ (green line), $T_{n, 10}^{M L}$ (blue line) and $T_{n, 10}^{M O}$ (red line).

\subsection{Practical Application}

We apply all tests from the simulation study in Section 5.3 to two real-world data examples. The first data set is from [39]. It was also analyzed by [13,40] with regard to the inverse Gaussianity hypothesis stated by [41]. The data are recalled in Table 4, where $n=46$ active repair times (in hours) for an airborne transceiver are provided. Table 5 shows the calculated value of each test statistic as well as the associated $p$-value. Each of the $p$-values is calculated using the classical parametric bootstrap approach used in Section 5 of [13]. Observing the $p$-values, none of the tests rejects the null hypothesis at a nominal significance level of $10 \%$. 
Table 4. Repair times (in hours) for airborne transceivers.

\begin{tabular}{cccccccccc}
\hline 0.2 & 0.3 & 0.5 & 0.5 & 0.5 & 0.5 & 0.6 & 0.6 & 0.7 & 0.7 \\
0.7 & 0.8 & 0.8 & 1.0 & 1.0 & 1.0 & 1.0 & 1.1 & 1.3 & 1.5 \\
1.5 & 1.5 & 1.5 & 2.0 & 2.0 & 2.2 & 2.5 & 2.7 & 3.0 & 3.0 \\
3.3 & 3.3 & 4.0 & 4.0 & 4.5 & 4.7 & 5.0 & 5.4 & 5.4 & 7.0 \\
7.5 & 8.8 & 9.0 & 10.3 & 22.0 & 24.5 & & & & \\
\hline
\end{tabular}

Table 5. Values of the test statistics and $p$-values for the repair time example.

\begin{tabular}{cccccc}
\hline Test & Test Statistic & $\boldsymbol{p}$-Value & Test & Test Statistic & $p$-Value \\
\hline$K S$ & 0.0682 & 0.9040 & $T_{46,0.1}^{M O}$ & 4.0310 & 0.6423 \\
$C M$ & 0.0327 & 0.8707 & $T_{46,1}^{M O}$ & 0.4870 & 0.6643 \\
$A D$ & 0.2195 & 0.8826 & $T_{46,10}^{M O}$ & 0.0223 & 0.6641 \\
$H K_{46,0}^{(1)}$ & 0.0137 & 0.9409 & $\widetilde{T}_{46,0.1}^{M L}$ & 0.0618 & 0.9445 \\
$H K_{46,0}^{(2)}$ & 0.0028 & 0.9608 & $\widetilde{T}_{46,1}^{M L}$ & 0.0320 & 0.8557 \\
$V G_{46}$ & 0.5770 & 0.7115 & $\widetilde{T}_{46,10}^{M L}$ & 0.0101 & 0.7923 \\
$B G_{46}$ & 0.0057 & 0.7579 & $\widetilde{T}_{46,0.1}^{M O}$ & 1.3230 & 0.6479 \\
$T_{46,0.1}^{M L}$ & 0.0949 & 0.9199 & $\widetilde{T}_{46,1}^{M O}$ & 0.4588 & 0.6577 \\
$T_{46,1}^{M L}$ & 0.0298 & 0.9073 & $\widetilde{T}_{46,10}^{M O}$ & 0.1227 & 0.6399 \\
$T_{46,10}^{M L}$ & 0.0020 & 0.8436 & & & \\
\hline
\end{tabular}

We also consider a second example, with data taken from [42] as analyzed by [13,43], where the inverse Gaussian distribution as an underlying model was again suggested by [44]. The $n=25$ recorded measurements correspond to precipitation (in inches) at Jug Bridge, Maryland. Table 6 provides the data itself, while Table 7 shows the values of the different test statistics as well as the associated $p$-value for each test. In contrast to the previous example, five of the tests reject the null hypothesis at a nominal significance level of $10 \%$. This casts some doubt as to whether or not the data in question were realized from an inverse Gaussian law. Note that the $p$-values associated with the newly proposed tests are substantially lower than those associated with most of the existing tests, the exception being the test of [8].

Table 6. Precipitation (in inches) at Jug Bridge, Maryland.

\begin{tabular}{lllll}
\hline 1.01 & 1.11 & 1.13 & 1.15 & 1.16 \\
1.17 & 1.17 & 1.20 & 1.52 & 1.54 \\
1.54 & 1.57 & 1.64 & 1.73 & 1.79 \\
2.09 & 2.09 & 2.57 & 2.75 & 2.93 \\
3.19 & 3.54 & 3.57 & 5.11 & 5.62 \\
\hline
\end{tabular}

Table 7. Values of the test statistics and $p$-values for the precipitation example.

\begin{tabular}{cccccc}
\hline Test & Test Statistic & $\boldsymbol{p}$-Value & Test & Test Statistic & $\boldsymbol{p}$-Value \\
\hline$K S$ & 0.0679 & 0.9127 & $T_{25,0.1}^{M O}$ & 1.9691 & 0.4380 \\
$C M$ & 0.0307 & 0.8944 & $T_{25,1}^{M O}$ & 0.2903 & 0.4357 \\
$A D$ & 0.2110 & 0.8968 & $T_{25,10}^{M, O}$ & 0.0123 & 0.3350 \\
$H K_{25,0}^{(1)}$ & 0.0121 & 0.9482 & $\widetilde{T}_{25,0.1}^{M L}$ & 0.3203 & 0.1080 \\
$H K_{25,0}^{(2)}$ & 0.0025 & 0.9630 & $\widetilde{T}_{25,1}^{M L}$ & 0.1796 & 0.0441 \\
$V G_{25}$ & 0.4314 & 0.7209 & $\widetilde{T}_{25,10}^{M L}$ & 0.0195 & 0.0584 \\
$B G_{25}$ & 0.0718 & 0.0123 & $\widetilde{T}_{25,0.1}^{M O}$ & 0.6804 & 0.4321 \\
$T_{25,0.1}^{M L}$ & 0.3216 & 0.1068 & $\widetilde{T}_{25,1}^{M O}$ & 0.3005 & 0.4356 \\
$T_{25,1}^{M L}$ & 0.1581 & 0.0632 & $\widetilde{T}_{25,10}^{M L}$ & 0.0825 & 0.3315 \\
$T_{25,10}^{M L}$ & 0.0029 & 0.0720 & & & \\
\hline
\end{tabular}




\section{Conclusions}

Since the global power function of any nonparametric test is flat on balls of alternatives except for alternatives coming from a finite-dimensional subspace-see [45] —it is known that there is no uniformly most powerful test for the considered problem. Nevertheless, the empirical powers presented above show that the newly proposed class of tests is very powerful compared to its competitors. In particular, the new test is more powerful than the other quite recent tests we considered, and it also outperforms the classical empirical distribution function-based tests.

Finally, we point out some interesting open questions for further research. In view of the data-driven method of choosing a tuning parameter proposed by [46], and refined by [47], for location scale families, it would be beneficial for the power of the tests to find an optimal choice of $a$, even though the procedures are not very sensitive to the choice of different tuning parameters. Since the inverse Gaussian distribution is not a location scale family, the current context does not lend itself to a simple solution. Another interesting question is to find characterizations similar to Theorem 1 for larger classes of distributions, i.e., supersets of $\mathcal{I} \mathcal{G}$, such as the generalized hyperbolic distributions, and apply a methodology similar to the present one to find powerful goodness-of-fit tests. These distributions have become popular in financial modelling and risk analysis. For initial results on Stein characterizations of the generalized hyperbolic distribution, see [48].

Author Contributions: The authors (J.S.A., S.B., B.E., J.V.) contributed equally. All authors have read and agreed to the published version of the manuscript.

Funding: This research received no external funding.

Institutional Review Board Statement: Not applicable.

Informed Consent Statement: Not applicable.

Data Availability Statement: Not applicable.

Acknowledgments: The work of J.S. Allison is based on research supported by the National Research Foundation (NRF). Any opinion, finding and conclusion or recommendation expressed in this material is that of the authors and the NRF does not accept any liability in this regard.

Conflicts of Interest: The authors declare no conflict of interest.

\section{References}

1. Bachelier, L. Théorie de la spéculation. Ann. Sci. L'École Norm. SupÉRieure 1900, 17, 21-86. [CrossRef]

2. Schroedinger, E. Zur Theorie der Fall- und Steigversuche an Teilchen mit Brownscher Bewegung. Phys. Z. 1915, 16, $289-295$.

3. Seshadri, V. The Inverse Gaussian Distribution: A Case Study in Exponential Families; Clarendon Press: Oxford, UK, 1993.

4. Tweedie, M.C.K. Statistical properties of inverse Gaussian distributions. I. Ann. Math. Stat. 1957, 28, 362-377. [CrossRef]

5. Tweedie, M.C.K. Statistical properties of inverse Gaussian distributions. II. Ann. Math. Stat. 1957, 28, 696-705. [CrossRef]

6. Seshadri, V. The Inverse Gaussian Distribution: Statistical Theory and Applications; Lecture Notes in Statistics; Springer: New York, NY, USA, 1999; Volume 137.

7. Chhikara, R.S.; Folks, J. L. The Inverse Gaussian Distribution: Theory, Methodology, and Applications; Marcel Dekker, Inc.: New York, NY, USA, 1989.

8. Baringhaus, L.; Gaigall, D. On an independence test approach to the goodness-of-fit problem. J. Multivar. Anal. 2015, 140, 193-208. [CrossRef]

9. Mudholkar, G.S.; Natarajan, R.; Chaubey, Y.P. A goodness-of-fit test for the inverse Gaussian distribution using its independence characterization. Sankhya Indian J. Stat. Ser. B 2001, 63, 362-374.

10. Ducharme, G.R. Goodness-of-fit tests for the inverse Gaussian and related distributions. TEST 2001, 10, 271-290. [CrossRef]

11. Nguyen, T.T.; Dinh, K.T. Exact EDF goodness-of-fit tests for inverse Gaussian distributions. Commun. Stat.-Simul. Comput. 2003, 32, 505-516. [CrossRef]

12. Gracia-Medrano, L.; O'Reilly, F.J. Transformations for testing the fit of the inverse-Gaussian distribution. Commun. Stat.-Theory Methods 2005, 33, 919-924. [CrossRef]

13. Henze, N.; Klar, B. Goodness-of-fit tests for the inverse Gaussian distribution based on the empirical Laplace transform. Ann. Inst. Stat. Math. 2002, 54, 425-444. [CrossRef]

14. Koutrouvelis, I.A.; Karagrigoriou, A. Cumulant plots and goodness-of-fit tests for the inverse Gaussian distribution. J. Stat. Comput. Simul. 2012, 82, 343-358. [CrossRef] 
15. Vexler, A.; Shan, G.; Kim, S.; Tsai, W.-M.; Tian, L.; Hutson, A.D. An empirical likelihood ratio based goodness-of-fit test for inverse Gaussian distributions. J. Stat. Plan. Inference 2011, 141, 2128-2140. [CrossRef]

16. Villaseñor, J.A.; González-Estrada, E. Tests of fit for inverse Gaussian distributions. Stat. Probab. Lett. 2015, 105, 189-194. [CrossRef]

17. Koudou, A.E.; Ley, C. Efficiency combined with simplicity: New testing procedures for generalized inverse Gaussian models. TEST 2014, 23, 708-724. [CrossRef]

18. Noughabi, H.A. An extensive power evaluation of some tests for the inverse Gaussian distribution. Commun. Stat.-Simul. Comput. 2017, 46, 5410-5422. [CrossRef]

19. Nikitin, Y.Y. Tests based on characterizations, and their efficiencies: A survey. Acta Comment. Univ. Tartu. Math. 2017, 21, 3-24. [CrossRef]

20. Koudou, A.E.; Ley, C. Characterizations of GIG laws: A survey. Probab. Surv. 2014, 11, 161-176. [CrossRef]

21. Ahsanullah, M. Characterizations of Univariate Continuous Distributions; Atlantis Studies in Probability and Statistics, Book 7; Atlantis Press: Paris, France, 2017.

22. Kagan, A.M.; Linnik, J.V.; Rao, C.R. Characterization Problems in Mathematical Statistics; Wiley Series in Probability and Mathematical Statistics; Wiley: New York, NY, USA, 1973.

23. Betsch, S.; Ebner, B. Fixed point characterizations of continuous univariate probability distributions and their applications. Ann. Inst. Stat. Math. 2021, 73, 31-59. [CrossRef]

24. Ley, C.; Reinert, G.; Swan, Y. Stein's method for comparison of univariate distributions. Probab. Surv. 2017, 14, 1-52. [CrossRef]

25. Baringhaus, L.; Ebner, B.; Henze, N. The limit distribution of weighted $L^{2}$-goodness-of-fit statistics under fixed alternatives, with applications. Ann. Inst. Stat. Math. 2017, 69, 969-995. [CrossRef]

26. Betsch, S.; Ebner, B. A new characterization of the Gamma distribution and associated goodness-of-fit tests. Metrika 2019, 82, 779-806. [CrossRef]

27. Meintanis, S.G. Testing skew normality via the moment generating function. Math. Methods Stat. 2010, 19, 64-72. [CrossRef]

28. Meintanis, S.G. A review of testing procedures based on the empirical characteristic function. S. Afr. Stat. J. 2016, 50, 1-14.

29. Johnson, N.L.; Kotz, S.; Balakrishnan, N. Continuous Univariate Distributions, 2nd ed.; Wiley: New York, NY, USA, 1995.

30. Bourguignon, M.; Saulo, H. On a new approach to estimate the shape parameter of the inverse Gaussian distribution. S. Afr. Stat. J. 2018, 52, 15-27.

31. Pandey, B.; Bandyopadhyay, P. Bayesian estimation of inverse gaussian distribution. arXiv 2012, arXiv:1210.4524.

32. Betró, B.; Rotondi, R. On Bayesian inference for the inverse Gaussian distribution. Stat. Probab. Lett. 1991, 11, 219-224. [CrossRef]

33. Ahmadini, A.A.H.; Javed, A.; Akhtar, S.; Chesneau, C.; Jamal, F.; Slshqaq, S.S.; Elgarhy, M.; Al-Marzouki, S.; Tahir, M.H.; Almutiry, W. Robust assessing the lifetime performance of products with inverse Gaussian distribution in Bayesian and classical setup. Hindawi 2021, 2021, 1024-1123. [CrossRef]

34. Chen, X.; White, H. Central limit and functional central limit theorems for Hilbert-valued dependent heterogeneous arrays with applications. Econom. Theory 1998, 14, 260-284. [CrossRef]

35. Le Cam, L. Locally asymptotically normal families of distributions. Univ. Calif. Publ. Stat. 1960, 3, 37-98.

36. Henze, N. Empirical-distribution-function goodness-of-fit tests for discrete models. Can. J. Stat. 1996, 24, 81-93. [CrossRef]

37. Giacomini, R.; Politis, D.N.; White, H. A warp-speed method for conducting Monte Carlo experiments involving bootstrap estimators. Econom. Theory 2013, 29, 567-589. [CrossRef]

38. Khati, C. A characterization of the inverse Gaussian distribution. Ann. Math. Stat. 1962, 33, 800-803. [CrossRef]

39. von Alven, W.H.E. Reliability Engineering by ARINC; Prentice Hall: Englewood Cliffs, NJ, USA, 1964.

40. Gunes, H.; Dietz, D.C.; Auclair, P.F.; Moore, A.H. Modified goodness-of-fit tests for the inverse Gaussian distribution. Comput. Stat. Data Anal. 1997, 24, 63-77. [CrossRef]

41. Chhikara, R.S.; Folks, J.L. The inverse Gaussian distribution as a lifetime model. Technometrics 1977, 19, 461-468. [CrossRef]

42. Ang, A.H.-S.; Tang, W.H. Probability Concepts in Engineering Planning and Design, Volume 1; Wiley: New York, NY, USA, 1975.

43. O'Reilly, F.J.; Rueda, R. Goodness of fit for the inverse Gaussian distribution. Can. J. Stat. 1992, 20, 387-397. [CrossRef]

44. Folks, J.L.; Chhikara, R.S. The inverse Gaussian distribution and its statistical application-A review. J. R. Stat. Soc. Ser. B (Methodol.) 1978, 40, 263-289. [CrossRef]

45. Janssen, A. Global power functions of goodness of fit tests. Ann. Stat. 2000, 28, 239-253. [CrossRef]

46. Allison, J.S.; Santana, L. On a data-dependent choice of the tuning parameter appearing in certain goodness-of-fit tests. J. Stat. Comput. Simul. 2015, 85, 3276-3288. [CrossRef]

47. Tenreiro, C. On the automatic selection of the tuning parameter appearing in certain families of goodness-of-fit tests. J. Stat. Comput. Simul. 2019, 89, 1780-1797. [CrossRef]

48. Gaunt, R.E. A Stein characterisation of the generalized hyperbolic distribution. ESAIM Probab. Stat. 2017, 21, 303-316. [CrossRef] 